\title{
Inhibition of interleukin-1/-stimulated matrix metalloproteinases via the controlled release of interleukin-1Ra from chitosan microspheres in chondrocytes
}

\author{
PANG-HU ZHOU ${ }^{1 *}$, BEI-LEI MA ${ }^{2 *}$, LEI SHI $^{3}$, TING XIE $^{4}$ and BO QIU ${ }^{1}$ \\ ${ }^{1}$ Department of Orthopedics, Renmin Hospital of Wuhan University, Wuhan, Hubei 430060; ${ }^{2}$ Department of Laboratory, \\ Qilu Hospital of Shandong University, Jinan, Shandong 250012; ${ }^{3}$ Department of Oncology, \\ Renmin Hospital of Wuhan University, Wuhan, Hubei 430060; ${ }^{4}$ Department of Women Health Care, \\ Hubei Women and Children's Hospital, Wuhan, Hubei 430070, P.R. China
}

Received December 19, 2013; Accepted July 21, 2014

DOI: $10.3892 / \mathrm{mmr} .2014 .2743$

\begin{abstract}
The aim of the study was to determine whether the controlled release of interleukin (IL)-1Ra from chitosan (CS) microspheres inhibits the IL-1 $\beta$-stimulated production of matrix metalloproteinases (MMPs) in chondrocytes. The CS-IL-1Ra microspheres were fabricated by an emulsification method using sodium tripolyphosphate as a crosslinker, and the controlled release of IL-1Ra was determined using an enzyme-linked immunosorbent assay. IL- $1 \beta$ was added to normal rat chondrocytes to stimulate MMP production. The chondrocytes were incubated with CS-IL-1Ra microspheres to assess its effects on IL-1 $\beta$-induced MMP expression. Chondrocyte proliferation and glycosaminoglycan (GAG) content were also determined. The mRNA expression and protein levels of IL-1 $\beta$, MMP-1, MMP-3 and MMP-13 were detected using reverse transcription-polymerase chain reaction and western blotting analyses, respectively. The IL-1Ra release kinetics were characterized by an initial burst release, which was reduced to a linear release over seven days. The mRNA expression levels and protein levels of IL-1 $\beta$, MMP-1, MMP-3 and MMP-13 were reduced compared with the control group. The present study demonstrated the chondroprotective properties of CS microspheres as a controlled release system carrying IL-1Ra, due to the ability of the system to downregulate the expression of osteoarthritis-associated matrix-degrading proteinases in chondrocytes.
\end{abstract}

Correspondence to: Dr Pang-Hu Zhou, Department of Orthopedics, Renmin Hospital of Wuhan University, 99 Ziyang Road, Wuhan, Hubei 430060, P.R. China

E-mail: zhoupanghu@gmail.com; zhoupanghu@126.com

${ }^{*}$ Contributed equally

Key words: chitosan, chondrocytes, metalloproteinases, interleukin-1 receptor antagonist, controlled-released

\section{Introduction}

Osteoarthritis (OA) is an age-related degenerative joint disease that at its late stage is characterized by extensive cartilage destruction and the loss of chondrocytes. The loss of the major components of the extracellular matrix (ECM) as a result of increased synthesis of matrix metalloproteases (MMPs) and aggrecanases (such as, ADAMTS), as well as reduced glycosaminoglycan (GAG) synthesis, has been well-documented during OA progression $(1,2)$. MMPs are zinc-containing, calcium-dependent proteinases, which collectively degrade all components of the extracellular matrix and have a critical role in intrinsic chondrocyte-mediated degenerative changes in the cartilage matrix in OA (3). MMPs are often expressed in chondrocytes in response to major inflammatory cytokines, including interleukin (IL)-1 $\beta$, which is produced by the synovium and other joint tissues (4). IL-1 $\beta$ has been thoroughly investigated, particularly its role as a key mediator of cartilage destruction in OA due to its ability to induce or upregulate the expression of proteinases, including MMPs, plasminogen activators and aggrecanases, and to downregulate the expression of endogenous proteinase inhibitors (for example, specific tissue inhibitors of metalloproteinases) (5-7). These products disrupt the metabolic balance of chondrocytes, resulting in a perturbation of the chondrocyte phenotype by reducing type II collagen expression $(5,6)$. This process induces specific degradation, and consequently the inhibition of the IL- $1 \beta$ pathway presents a promising means to prevent cartilage degradation during OA pathogenesis. One of the major endogenous inhibitors of the IL-1 pathway is the IL-1 receptor antagonist (IL-1Ra). IL-1Ra is a well-known anti-inflammatory cytokine that is a member of the IL-1 family. IL-1Ra binds to the IL-1RI receptor with similar specificity and affinity but does not activate downstream signals $(8,9)$. In addition, IL-1Ra has been used as a gene therapeutic strategy in the treatment of OA, suggesting that it may also be appropriate for the treatment of early-stage, cytokine-mediated cartilage degeneration $(10,11)$.

With the aim of enhancing the potential role of IL-Ra in the treatment of OA, it was proposed that the encapsulation of this macromolecule in select microspheres capable of positively 
interacting with chondrocytes, where the macromolecule may be internalized by chondrocytes and released as encapsulated IL-1Ra in a controlled manner.

Chitosan (CS) is a natural copolymer of D-glucosamine and $\mathrm{N}$-acetylglucosamine, which is derived from chitin and is structurally similar to GAGs. Chitosan has been reported to be non-toxic and bioabsorbable (12) and is known to promote wound healing (13) and contribute to the maintenance of the chondrogenic phenotype (14), particularly in its morphology. Furthermore, CS has been widely used in various nanocarriers and is considered to enhance drug absorption through tight junctions via a paracellular route by direct interactions of the cationic polymer molecule with negatively charged cellular membranes. Numerous studies have successfully applied the use of CS in drug $(15,16)$ and DNA delivery $(17,18)$, and tissue engineering (19).

The present study aimed to combine the virtues of CS in the development of IL-Ra-loaded microspheres, as a potential novel drug-release system for OA treatment. The interaction between these microspheres and chondrocytes, as well as their potential in protecting cartilage, was evaluated.

\section{Materials and methods}

Materials. CS (molecular weight, $150 \mathrm{kDa}$; deacetylation, 95\%) and sodium tripolyphosphate (STPP) were obtained from Sigma-Aldrich (St. Louis, MO, USA). Recombinant rat (rr) IL-1Ra was purchased from PeproTech (Rocky Hill, NJ, USA). Trypsinase, collagenase II and Dulbecco's modified Eagle's medium (DMEM)/F12 were purchased from Gibco-BRL (Carlsbad, CA, USA). All of the other chemicals used in the present study were of the highest available commercial grade.

Microsphere preparation and characterization. CS microspheres were prepared according to an emulsion ionic cross-linking method modified from previously described methods (20). The microspheres were spontaneously obtained via inotropic gelation between the positively charged amino groups of CS and the negatively charged amino groups of TPP and IL-1Ra proteins. Under magnetic stirring at room temperature, $3.5 \mathrm{ml}$ of a mixture of an aqueous solution of TPP $(0.06 \mathrm{mg} / \mathrm{ml})$ and IL-1Ra was added to $3.5 \mathrm{ml}$ of a solution of CS (1\%, w/v, pH 5.0). Magnetic stirring was maintained for $10 \mathrm{~min}$ for complete stabilization of the system. Next, the microspheres were transferred to Eppendorf tubes and isolated by centrifugation in a glycerol bed $\left(16,000 \times \mathrm{g}, 30 \mathrm{~min}, 25^{\circ} \mathrm{C}\right)$. The supernatants were collected and the microspheres were then resuspended in ultrapure water by shaking on a vortex mixer (Beijing Donglinchangsheng Biotechnology Co., Ltd., Beijing, China). Next, the microspheres were centrifuged from the fixed volumes of microsphere suspension $(16,000 \mathrm{x} \mathrm{g}$, $30 \mathrm{~min}, 25^{\circ} \mathrm{C}$ ) without a glycerol bed. The supernatants were discarded and the sediments were freeze-dried. CS microspheres were prepared, and the sizes and shapes of the microspheres were examined using a scanning electron microscope (JSM-6510; Jeol, Ltd., Tokyo, Japan).

In vitro release profiles. Approximately $30 \mathrm{mg}$ microspheres were placed in $1.5 \mathrm{ml}$ microcentrifuge tubes containing $1 \mathrm{ml}$ phosphate-buffered saline (PBS), and incubated at $37^{\circ} \mathrm{C}$ in a shaking bath at $108 \mathrm{x} \mathrm{g}$ at the indicated time periods for up to seven days. Periodically, the microsphere suspension was centrifuged, at $10,000 \mathrm{x}$ g for $10 \mathrm{~min}$, and the supernatant was collected for IL-1Ra analysis. The tubes obtained were resuspended in fresh PBS. Samples were then assayed for the IL-1Ra concentration using enzyme-linked immunosorbent assay kits (PeproTech) according to the manufacturer's instructions.

Chondrocyte isolation and culture conditions. Eight-day-old Sprague Dawley rats were obtained from the Experimental Animal Center of Wuhan University (Wuhan, China) and were housed under standard conditions (temperature, $21 \pm 1^{\circ} \mathrm{C}$; humidity, 55-60\%) with food and water available ad libitum. The care and use of the animals followed the recommendations and guidelines of the National Institutes of Health and were approved by the Wuhan University Animal Care and Use Committee (Wuhan, China).

Rat cartilage was isolated from the knee joints and placed into PBS. Briefly, the cartilage tissues were cut into small pieces $\left(<1 \mathrm{~mm}^{3}\right)$ and digested in $0.2 \%$ trypsin and $0.2 \%$ type II collagenase for $30 \mathrm{~min}$ and $2 \mathrm{~h}$, respectively. Following washing twice with DMEM, the released cells were cultured in DMEM/F12 medium supplemented with $10 \%$ fetal bovine serum (FBS) and antibiotics (1\% v/v penicillin/streptomycin) at $37^{\circ} \mathrm{C}$ with $5 \% \mathrm{CO}_{2}$. The cell viability was determined using a cell viability analyzer (viability, $>90 \%$; Beckman Coulter, Inc., Pasadena, CA, USA). The primary cells were maintained in a monolayer culture throughout the study. After the cells reached $70-80 \%$ confluence, the medium was changed to DMEM/F12 without $10 \%$ FBS and antibiotics for $6 \mathrm{~h}$. IL-1 $\beta$ $(10 \mathrm{ng} / \mathrm{ml})$ was then added to the culture medium without rinsing for an additional $48 \mathrm{~h}$. The cultured chondrocytes were divided into four groups: (i) Blank group, with no treatment; (ii) controls, treated with IL-1 $\beta$ only; (iii) treated with IL-1 $\beta$ and CS microspheres; and (iv) treated with IL-1 $\beta$ and CS-IL-1Ra microspheres. All of the cells were cultured in DMEM/F12, containing $10 \%$ FBS without antibiotics, and incubated for $4 \mathrm{~h}$ after the treatment. Four samples were taken from each group and each experiment was repeated five times. Four samples were taken from each group and each experiment was repeated five times.

Cell proliferation and GAG synthesis. Following $72 \mathrm{~h}$ of co-culture, the microsphere solution was discarded and fresh DMEM media containing $0.5 \mathrm{mg} / \mathrm{ml} \mathrm{3-(4,5-dimethylthi-}$ azol-2-yl)-2, 5-dippphenyltetrazolium bromide was added to the chondrocytes and incubated at $37^{\circ} \mathrm{C}$ with $5 \% \mathrm{CO}_{2}$ for $4 \mathrm{~h}$. The resulting precipitate was dissolved in dimethylsulfoxide and the absorbance was measured at $570 \mathrm{~nm}$ using a microplate reader (Shimadzu, Kyoto, Japan).

The dimethylmethylene blue (DMMB) spectrophotometric assay was used to determine the GAG contents in the cell supernatants. The cell supernatants were digested in $0.5 \mathrm{mg} / \mathrm{ml}$ papain for $2 \mathrm{~h}$ at $65^{\circ} \mathrm{C}$. Following digestion, the supernatants were centrifuged at 1,500 x $\mathrm{g}$ for $8 \mathrm{~min}$. Next, the DMMB solution (Sigma, St. Louis, MO, USA) was added to the digested cell supernatants, and the absorbance was measured at $525 \mathrm{~nm}$ using a UV-1601 spectrophotometer (Shimadzu). A standard curve was derived from the mixed-isomer shark chondroitin sulfate and the GAG content was calculated. 
Table I. Effects of microphere treatment on IL-1 $\beta$-stimulated chondrocytes.

\begin{tabular}{|c|c|c|c|c|}
\hline Group & Blank & $10 \mathrm{ng} / \mathrm{ml} \mathrm{IL-1 \beta}$ & $\begin{array}{c}10 \mathrm{ng} / \mathrm{ml} \mathrm{IL-}-1 \beta \\
+\mathrm{CS}\end{array}$ & $\begin{array}{c}10 \mathrm{ng} / \mathrm{ml} \mathrm{IL-} \beta \\
+\mathrm{CS}-\mathrm{IL}-1 \mathrm{Ra}\end{array}$ \\
\hline GAG content $(\mu \mathrm{g} / \mathrm{ml})$ & $12.53 \pm 1.24$ & $4.09 \pm 1.67^{\mathrm{a}}$ & $7.38 \pm 0.51^{\mathrm{b}}$ & $10.43 \pm 0.82^{b}$ \\
\hline Cell proliferation & $0.92 \pm 0.02$ & $0.39 \pm 0.11^{\mathrm{a}}$ & $0.62 \pm 0.07^{b}$ & $0.81 \pm 0.04^{\mathrm{b}, \mathrm{c}}$ \\
\hline
\end{tabular}

Statistical results represent the means \pm standard deviation of five separate experiments. ${ }^{a} \mathrm{P}<0.05$ vs. the blank group; ${ }^{\mathrm{b}} \mathrm{P}<0.05$ vs. the control group; ${ }^{\mathrm{C}}<0.05$ vs. the $\mathrm{CS}$ plus IL- $1 \beta$ treated group. IL-1 $\beta$, interluekin- $1 \beta$; GAG, glycosaminoglycan; CS, chitosan.

Reverse transcription-polymerase chain reaction $(R T-P C R)$ for $I L-1 \beta, M M P-1, M M P-3$ and $M M P-13$. Total RNA was extracted from chondrocytes using TRIzol ${ }^{\circledR}$ (Invitrogen Life Technologies, Carlsbad, CA, USA) reagent according to the manufacturer's instructions. Total RNA was quantified using a spectrophotometer (DU730; Beckman Coulter, Inc, Brea, CA, USA) at $260 \mathrm{~nm}$ and the purity was assessed by determining the ratio of A260/A280. All of the samples had ratios $>1.75$. Reverse transcription was performed at $42^{\circ} \mathrm{C}$ for $60 \mathrm{~min}$ in a total volume of $25 \mu \mathrm{l}$ under standard conditions with $1 \mu \mathrm{g}$ total RNA and $0.5 \mu \mathrm{g}$ random hexamer primers (Takara Biotechnology Co., Ltd., Dalian, China). The cDNA was amplified using PCR with the following primer sets: Forward: 5'-GAGCCTGTCATCTTCGAAACG-3' and reverse: 5'-GCACGGGTGCGTCACA-3' for IL-1 $\beta$; forward: 5'-GCTAACCTTTGATGCTATAACTACGA-3' and reverse: 5'-TTTGTGCGCATGTAGAATCT-3' for MMP-1; forward: 5'-CAAA ACATATTTCTTTGTAGAGGACAA-3' and reverse: 5'-TTCAGCTATTTGCTTGGGAAA-3' for MMP-3; and forward: 5'-TGGTCCA GGAGATGAAGACC-3' and reverse: 5'-TGGCATCAAGGGATAAGGAA-3' for MMP-13. As a control, GAPDH was amplified in parallel using the following primers: Forward: 5'-ACCACAGTCCATGCCATCAC-3' and reverse: 5'-TCCACCACCCTGTTGCTGTA-3'. The PCR amplification was performed under standard conditions using $25 \mu \mathrm{l}$ reaction mixture consisting of $0.5 \mu \mathrm{l}$ of $10 \mathrm{~mm} \mathrm{dNTP,} 2.5 \mu \mathrm{l}$ of $10 \mathrm{X}$ buffer (containing $\mathrm{Mg}^{2+}$ ), $1 \mu \mathrm{l}$ upstream primer $(50 \mu \mathrm{g} / \mathrm{ml}), 1 \mu \mathrm{l}$ downstream primer $(50 \mu \mathrm{g} / \mathrm{ml}), 4 \mu \mathrm{l} \mathrm{cDNA}$ and 1 unit Taq enzyme. The reaction conditions consisted of 30 cycles of denaturation at $94^{\circ} \mathrm{C}$ for $60 \mathrm{sec}$, annealing at $53^{\circ} \mathrm{C}$ for $60 \mathrm{sec}$, extension at $72^{\circ} \mathrm{C}$ for $60 \mathrm{sec}$ and a final extension at $72^{\circ} \mathrm{C}$ for $5 \mathrm{~min}$. The PCR amplification products were analyzed on a $1.5 \%$ agarose gel stained with ethidium bromide and measured semiquantitatively using the Bio-Rad Multi-Analyst system (Bio-Rad Laboratories, Hercules, CA, USA).

Western blotting analyses for IL-1 $\beta$ MMP-1, MMP-3 and $M M P-13$. The proteins were extracted from harvested chondrocytes. The protein concentrations were determined using the Bicinchoninic Acid Protein Assay kit (Beyotime Institute of Biotechnology, Haimen, China). Following adjusting to equal quantities of proteins (50 $\mu 1$ protein/lane), the proteins were separated using sodium dodecyl sulfate-polyacrylamide gel electrophoresis (SDS-PAGE) under reducing conditions and then transferred onto polyvinylidene difluoride membranes
(Bio-Rad Laboratories). The membranes were blocked in PBS, pH 7.4, containing 5\% non-fat dry milk, and initially incubated with rabbit polyclonal anti-IL-1 $\beta$ (EMD Millipore, Billerica, MA, USA), rabbit polyclonal MMP-1 (EMD Millipore), rabbit monoclonal MMP-3 (Abcam, Cambridge, MA, USA) and rabbit polyclonal MMP-13 (Abcam). Next, the membranes were incubated with horseradish peroxidase-conjugated secondary antibodies (goat anti-rabbit IgG; Santa Cruz Biotechnology Inc., Dallas, TX, USA), followed with visualization using an enhanced chemiluminescence kit (PerkinElmer, Waltham, MA, USA).

Statistical analysis. SPSS 19.0 (SPSS, Inc., Armonk, NY, USA) software was applied for data analysis. The data are presented as the mean \pm standard deviation of five independent experiments. Each experimental condition was performed in triplicate wells, where the replicates from each culture were averaged and combined as one value for analysis. Significant differences among the mean values of multiple groups were evaluated using one-way analysis of variance and Student-Newman-Keuls q-test. $\mathrm{P}<0.05$ was considered to indicate a statistically significant difference.

\section{Results}

Characterization of microspheres. The CS-IL-1Ra microspheres were fabricated using the emulsion ionic cross-linking method. The ability of CS to form a gel following contact with polyanions (TPP and IL-1Ra) by promoting the inter- and intramolecular linkage enables the formation of the microspheres. The morphology of the microspheres is demonstrated in Fig. 1. The SEM demonstrated that all of the resulting microspheres were distributed uniformly (Fig. 1A). The microspheres were spherical and the surfaces were nearly smooth. The surfaces of the microspheres appeared to be porous (Fig. 1B), and their diameters ranged from 7 to $18 \mu \mathrm{M}$. Compared with the CS-IL-1Ra group (Fig. 1C), the mean size of the microspheres in the CS group was smaller (Fig. 1D).

In vitro release profiles. The release kinetics of IL-1Ra from the CS microspheres are shown in Fig. 2. In the CS-IL-1Ra microspheres, the IL-1Ra protein was released slowly. The release kinetics were monitored over seven days, with a final release of $\sim 80 \%$ of IL-1Ra within seven days of incubation. The IL-1Ra release kinetics were characterized by an initial burst release, which was reduced to a linear release. 
Table II. mRNA expression of MMP-1, $-3,-13$ and IL-1 $\beta$ in chondrocytes.

\begin{tabular}{|c|c|c|c|c|}
\hline Group & Blank & $10 \mathrm{ng} / \mathrm{ml} \mathrm{IL-1} \beta$ & $\begin{array}{c}10 \mathrm{ng} / \mathrm{ml} \mathrm{IL-}-1 \beta \\
+\mathrm{CS}\end{array}$ & $\begin{array}{c}10 \mathrm{ng} / \mathrm{ml} \mathrm{IL}-1 \beta \\
+\mathrm{CS}-\mathrm{IL}-1 \mathrm{Ra}\end{array}$ \\
\hline MMP-1 & $0.34 \pm 0.02$ & $1.66 \pm 0.09^{a}$ & $0.96 \pm 0.07^{\mathrm{b}}$ & $0.58 \pm 0.05^{\mathrm{b}}$ \\
\hline MMP-3 & $0.47 \pm 0.11$ & $1.45 \pm 0.18^{\mathrm{a}}$ & $0.82 \pm 0.08^{b}$ & $0.63 \pm 0.11^{\mathrm{b}}$ \\
\hline MMP-13 & $0.41 \pm 0.08$ & $0.83 \pm 0.12^{\mathrm{a}}$ & $0.75 \pm 0.09^{b}$ & $0.68 \pm 0.08^{b}$ \\
\hline IL-1 $\beta$ & $0.26 \pm 0.04$ & $1.40 \pm 0.15^{\mathrm{a}}$ & $0.73 \pm 0.07^{\mathrm{b}}$ & $0.55 \pm 0.06^{\mathrm{b}}$ \\
\hline
\end{tabular}

Statistical results are represented as the means \pm standard deviation of five separate experiment. ${ }^{\mathrm{a}} \mathrm{P}<0.05$ vs. the blank group and ${ }^{\mathrm{b}} \mathrm{P}<0.05$ vs. the control group. MMP, matrix metalloproteinase; CS, chitosan; IL-1 $\beta$, interleukin-1 $\beta$.

A

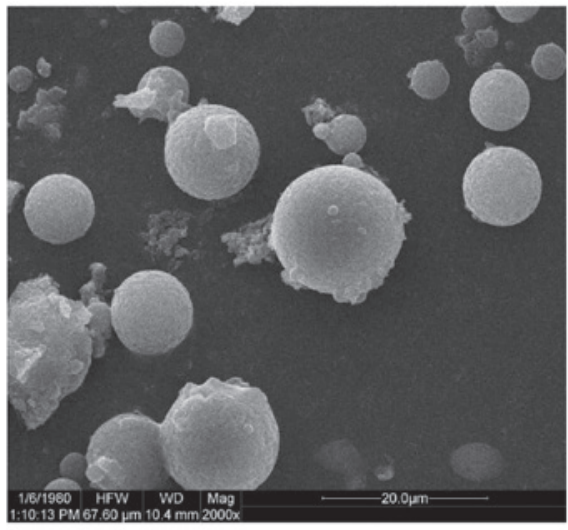

C

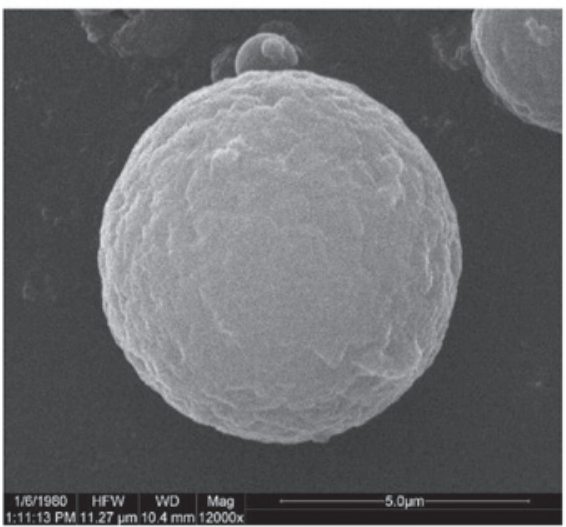

B

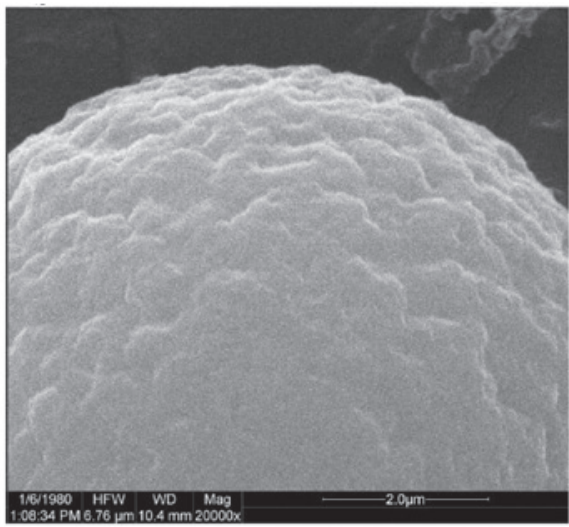

D

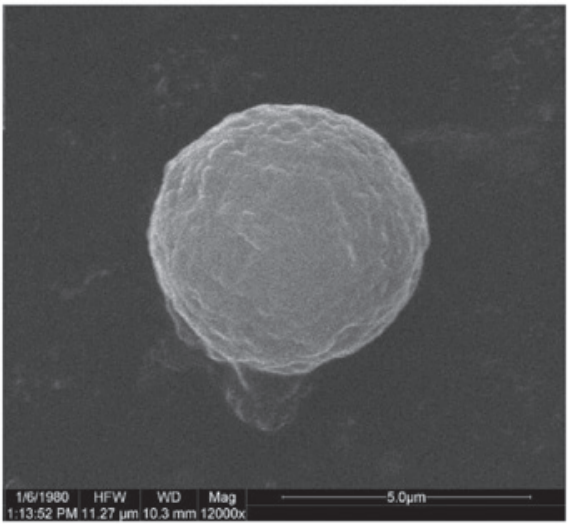

Figure 1. (A) Image of various microspheres: (B) Surface image of a microsphere; (C) Image of CS-IL-1Ra microspheres; (D) Image of CS microspheres.

Assay of cell proliferation. The monolayer cultured chondrocyte proliferation was significantly increased in the CS-IL-1Ra group and the CS group compared with the control group. Furthermore, chondrocyte proliferation was significantly higher in the CS-IL-1Ra group compared with the CS group. The results are demonstrated in Table I.

GAG contents in cell supernatants. Compared with the control group, the GAG contents were higher in the CS-IL-1Ra $(10.43 \pm 0.82 \mu \mathrm{g} / \mathrm{ml})$ and CS $(7.38 \pm 0.51 \mu \mathrm{g} / \mathrm{ml})$ groups, while the GAG content in the CS-IL-1Ra group was marginally increased in the CS group, as shown in Table I.

RT-PCR for IL-1 $\beta, M M P-1, M M P-3$ and MMP-13. A significant decrease in the expression of IL-1 $\beta$, MMP-1, MMP-3 and MMP-13 in all of the experimental groups compared with the control group was observed as demonstrated in Table II and Fig. 3.

Western blotting analyses for $I L-1 \beta, M M P-1, M M P-3$ and $M M P-13$. The expression of IL-1 $\beta$, MMP-1, MMP-3 and MMP-13 was decreased in the CS-IL-1Ra and CS-IL-1Ra groups compared with the control group. Compared with the CS group, the CS-IL-1Ra group exhibited a greater decrease in the expression of IL-1 $\beta$, MMP-1, MMP-3 and MMP-13 (Fig. 4).

\section{Discussion}

The aim of the present study was to clarify the feasibility of using novel microspheres containing IL-1Ra as a drug release 


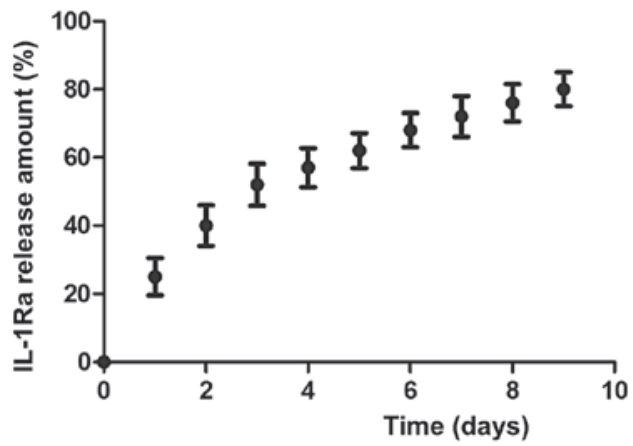

Figure 2. Release kinetics of CS-IL-1Ra microspheres. CS, chitosan; IL-1Ra, IL-1 receptor antagonist.
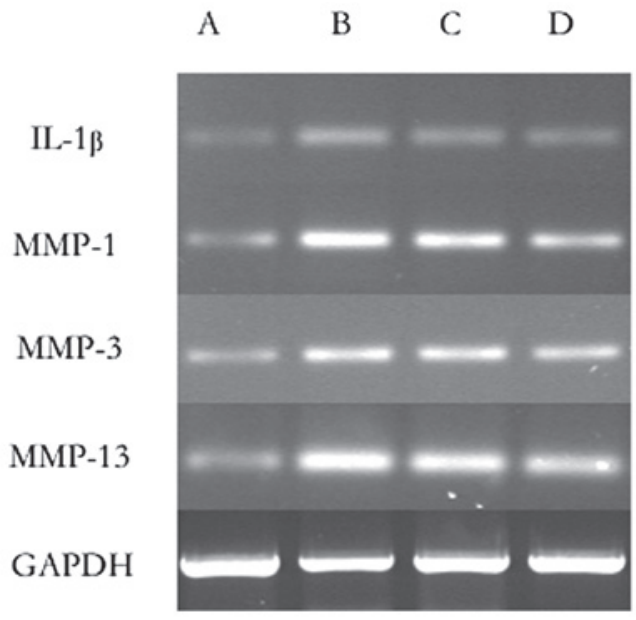

Figure 3. mRNA expression of MMPs and IL-1 $\beta$. MMP, matrix metalloproteinase; IL-1 $\beta$, interleukin- $1 \beta$.

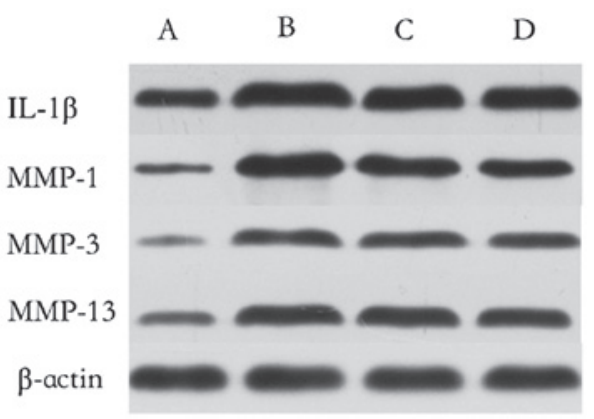

Figure 4. Western blot analyses for MMPs and IL-1 $\beta$. MMP, matrix metalloproteinase; IL-1 $\beta$, interleukin-1 $\beta$.

system to prevent the degeneration of articular cartilage. The results demonstrated that proliferation and ECM production, including GAGs, in chondrocytes cultured with microspheres evolved normally compared with the control group. The IL-1Ra controlled-release method was long-acting and efficacious. The expression of inflammatory cytokine production was shown to be decreased. On the basis of previous studies and the results of the present study, it was concluded that the controlled-release of IL-1Ra from CS microspheres demonstrated potential as a novel method of OA treatment.
GAG is a type of polysaccharide, which together with collagen type II forms the main component of the cartilage matrix. The secretion of GAGs in in vitro cultured chondrocytes is regarded as an indication that the chondrocyte phenotype is maintained (21). The present study demonstrated that microspheres were able to inhibit IL-1 $\beta$-stimulated production of MMP-1, MMP-3 and MMP-13 in chondrocytes. Therefore, treatment with microspheres may prevent the IL-1 $\beta$-induced breakdown of GAGs and maintain the multi-capacity of chondrocytes in cartilage by blocking MMPs, resulting in the delay of OA progression. MMPs destroy arch-like cartilage structures, which results in disruption of the elastic tissue via the degradation of collagen II, elastic fibers, proteoglycans and damage to the molecular sieve function (3). Such an effect renders articular cartilage more sensitive to catabolic enzymes. As revealed in Table II, the expression of MMPs was decreased, while GAG production was significantly increased in the CS-IL-1Ra and CS groups, but the CS-IL-1Ra group showed a greater decrease. These data indicated that IL-1Ra was key in decreasing the production of MMPs in microspheres. Furthermore, IL-1Ra is a naturally occurring inflammatory inhibitor protein, which is produced by corneal epithelial cells, monocytes, neutrophils, macrophages and fibroblasts. It inhibits the activity of IL- $1 \alpha$ and IL- $1 \beta$ by competitively inhibiting binding to type I and type II receptors, and subsequently blocking the IL-1 $\beta$-mediated downstream inflammatory signaling pathway (22). In addition, IL-1Ra may be chondroprotective as it inhibits IL-1 $\beta$. It has been reported that IL-1 $\beta$ may promote the destruction of the cartilage matrix via an increase in inflammatory cytokine production by chondrocytes, including IL-8, TNF- $\alpha$ and IL-1 $\beta$ (23); a positive feedback mechanism. Simultaneously, the expression of these inflammatory cytokines was decreased by the addition of IL-1Ra (24). It was also reported that human osteoarthritis-affected chondrocytes transfected with IL-1Ra in vivo demonstrated resistance to IL-1 $\beta$-mediated damage, thus indicating that IL-1 $\beta$ released by osteoarthritis-affected chondrocytes is an important in the pathophysiology of osteoarthritis (23). In the present study, the CS-IL-1Ra microspheres effectively suppressed the expression of IL-1 $\beta$. The suppression of inflammatory cytokine activity within the joint may also be an important mechanism underlying the clinical activity of microspheres in OA treatment. Therefore, the results of the present study demonstrate that IL-1Ra with CS may be a potential treatment strategy for patients with OA.

A considerable limitation of this study is that the results were derived from an in vitro experimental model. Whether CS microspheres loaded with IL-1Ra attenuate the degeneration of articular cartilage may only be determined by performing in vivo experiments. Although CS have been observed to evoke a minimal foreign body response in a number of studies $(25,26)$, the biocompatibility or immunological reaction of these materials to joint tissue remains unclear and the process of degradation or absorption of these materials in the articular environment remains unknown. This is the next challenge in validating our hypothesis.

Despite the limitation mentioned above, the data derived from this study suggest promise in the utilization of CS microspheres as IL-1Ra carriers for the treatment of OA. CS microspheres, as a novel drug-release system, may be applied 
to cartilage tissue engineering. On the basis of these results, bioactive substances, such as cell growth factors, basic fibroblast growth factor and insulin-like growth factor-I, may also be loaded into microspheres to enhance chondrogenesis in $\mathrm{OA}$ treatment.

\section{Acknowledgements}

The present study was supported by the National Natural Science Foundation (grant no. 81071494), the Natural Science Foundation of Hubei Province (grant no. 2011CHB021) and Young Scientists Foundation of Health Department of Hubei Province (grant no. QJX2012-12) in China. All of the authors listed in this manuscript actively participated in designing the experiments, analyzing and interpreting the data and helping to enhance the intellectual content of this manuscript.

\section{References}

1. Hardingham T: Extracellular matrix and pathogenic mechanisms in osteoarthritis. Curr Rheumatol Rep 10: 30-36, 2008.

2. Lorenzo P, Bayliss MT and Heinegård D: Altered patterns and synthesis of extracellular matrix macromolecules in early osteoarthritis. Matrix Biol 23: 381-391, 2004.

3. Suzuki M, Hashizume M, Yoshida H, Shiina M and Mihara M: IL-6 and IL-1 synergistically enhanced the production of MMPs from synovial cells by up-regulating IL-6 production and IL-1 receptor I expression. Cytokine 51: 178-183, 2010.

4. Martel-Pelletier J, Boileau C, Pelletier JP and Roughley PJ: Cartilage in normal and osteoarthritis conditions. Best Pract Res Clin Rheumatol 22: 351-384, 2008.

5. Billinghurst $\mathrm{RC}, \mathrm{Wu} \mathrm{W}$, Ionescu $\mathrm{M}$, et al: Comparison of the degradation of type II collagen and proteoglycan in nasal and articular cartilages induced by interleukin-1 and the selective inhibition of type II collagen cleavage by collagenase. Arthritis Rheum 43: 664-672, 2000.

6. Yasuda T and Poole AR: A fibronectin fragment induces type II collagen degradation by collagenase through an interleukin-1-mediated pathway. Arthritis Rheum 46: 138-148, 2002.

7. Wheaton AJ, Borthakur A, Shapiro EM, et al: Proteoglycan loss in human knee cartilage: quantitation with sodium MR imaging - feasibility study. Radiology 231: 900-905, 2004.

8. Gabay C, Lamacchia C and Palmer G: IL-1 pathways in inflammation and human diseases. Nat Rev Rheumatol 6: 232-241, 2010

9. Gouze E, Pawliuk R, Gouze JN, et al: Lentiviral-mediated gene delivery to synovium: potent intra-articular expression with amplification by inflammation. Mol Ther 7: 460-466, 2003.

10. Morisset S, Frisbie DD, Robbins PD, Nixon AJ and McIlwraith CW: IL-1ra/IGF-1 gene therapy modulates repair of microfractured chondral defects. Clin Orthop Relat Res 462: 221-228, 2007.
11. Chen B, Qin J, Wang H, Magdalou J and Chen L: Effects of adenovirus-mediated bFGF, IL-1Ra and IGF-1 gene transfer on human osteoarthritic chondrocytes and osteoarthritis in rabbits. Exp Mol Med 42: 684-695, 2010.

12. Sobol M, Bartkowiak A, de Haan B and de Vos P: Cytotoxicity study of novel water-soluble chitosan derivatives applied as membrane material of alginate microcapsules. J Biomed Mater Res A 101: 1907-1914, 2013.

13. Lih E, Lee JS, Park KM and Park KD: Rapidly curable chitosan-PEG hydrogels as tissue adhesives for hemostasis and wound healing. Acta Biomater 8: 3261-3269, 2012.

14. Chang SH, Hsiao YW and Lin HY: Low-frequency electromagnetic field exposure accelerates chondrocytic phenotype expression on chitosan substrate. Orthopedics 34: 20, 2011.

15. Haupt JL, Frisbie DD, McIlwraith CW, et al: Dual transduction of insulin-like growth factor-I and interleukin-1 receptor antagonist protein controls cartilage degradation in an osteoarthritic culture model. J Orthop Res 23: 118-126, 2005.

16. Lee JE,Kim KE,Kwon IC, et al: Effects of the controlled-released TGF-beta1 from chitosan microspheres on chondrocytes cultured in a collagen/chitosan/glycosaminoglycan scaffold. Biomaterials 25: 4163-4173, 2004.

17. Wu H, Wang S, Fang $\mathrm{H}$, et al: Chitosan-polycaprolactone copolymer microspheres for transforming growth factor- $\beta 1$ delivery. Colloids Surf B Biointerfaces 82: 602-8, 2011.

18. Zhao J, Fan X, Zhang Q, et al: Chitosan-plasmid DNA nanoparticles encoding small hairpin RNA targeting MMP-3 and -13 to inhibit the expression of dedifferentiation related genes in expanded chondrocytes. J Biomed Mater Res A 102: 373-380, 2014.

19. Suh JK and Matthew HW: Application of chitosan-based polysaccharide biomaterials in cartilage tissue engineering: a review. Biomaterials 21: 2589-2598, 2000.

20. Zeng W, Huang J, Hu X, et al: Ionically cross-linked chitosan microspheres for controlled release of bioactive nerve growth factor. Int J Pharm 421: 283-290, 2011.

21. Simonaro CM: Cartilage and chondrocyte pathology in the mucopolysaccharidoses: The role of glycosaminoglycan-mediated inflammation. J Pediatr Rehabil Med 3: 85-88, 2010.

22. Jacques C, Gosset M, Berebaum F and Gabay C: The role of IL-1 and IL-1Ra in joint inflammation and cartilage degradation. Vitam Horm 74: 371-403, 2006

23. Baragi VM, Renkiewicz RR, Jordan H, et al: Transplantation of transduced chondrocytes protects articular cartilage from interleukin 1-induced extracellular matrix degradation. J Clin Invest 96: 2454-2460, 1995.

24. Aida Y, Maeno M, Suzuki N, et al: The effect of IL-1beta on the expression of inflammatory cytokines and their receptors in human chondrocytes. Life Sci 79: 764-771, 2006.

25. Pattani A, Patravale VB, Panicker L and Potdar PD: Immunological effects and membrane interactions of chitosan nanoparticles. Mol Pharm 6: 345-352, 2009.

26. Song S, Zhou F, Nordquist RE, et al: Glycated chitosan as a new non-toxic immunological stimulant. Immunopharmacol Immunotoxicol 31: 202-208, 2009. 